\title{
SOME REMARKS ON PRAMARTS AND MILS
}

\author{
by ZHEN-PENG WANG $†$ and XING-HONG XUE
}

(Received 10 January, 1992)

1. Notations and summary. Let $F$ be a Banach space, $(\Omega, \mathscr{F}, P)$ a fixed probability space, $D$ a directed set filtering to the right with the order $\leq$, and $\left(\mathscr{F}_{t}, D\right)$ a stochastic basis of $\mathscr{F}$, i.e. $\left(\mathscr{F}_{t}, D\right)$ is an increasing family of sub- $\sigma$-algebras of $\mathscr{F}_{:}: \mathscr{F}_{s} \subset \mathscr{F}_{t}$ for any $s, t \in D$ and $s \leq t$. Throughout this paper, $\left(X_{t}\right)$ is an $F$-valued, (F्F $)$-adapted sequence, i.e. $X_{t}$ is $\mathscr{F}_{t}$-measurable, $t \in D$. We also assume that $X_{t} \in L^{1}$, i.e. $\int\left\|X_{t}\right\|<\infty$. We use $I(H)$ to denote the indicator function of an event $H$. Let $\infty$ be a such element: $t<\infty, t \in D$, $\bar{D}=D \cup \infty$, and $\mathscr{F}_{\infty}=\sigma\left(\bigcup_{t \in D} \mathscr{F}_{t}\right)$. A stopping time is a map $\tau: \Omega \rightarrow \bar{D}$ such that $(\tau \leq t) \in \mathscr{F}_{t}$, $t \in D$. A stopping time $\tau$ is called simple (countable) if it takes finitely (countably) many values in $D(\bar{D})$. Let $T$ and $T_{c}$ be the sets of simple and countable stopping times respectively and $T_{f}=\left\{\tau \in T_{c}: \tau<\infty\right.$ a.s. $\}$. Clearly, $(T, \leq)$ and $\left(T_{f}, \leq\right)$ are directed sets filtering to the right. For $\tau \in T_{c}$, let

$$
\mathscr{F}_{\tau}=\left\{H \in \mathscr{F}: H(\tau=t) \in \mathscr{F}_{t} \text { for all } t \in D\right\}, \quad X_{\tau}=\sum_{t \in D} X_{t} I(\tau=t),
$$

and

$$
\begin{gathered}
\mathscr{B}=\left\{\left(X_{t}\right): \sup _{\tau \in T} \int\left\|X_{\tau}\right\|<\infty\right\}, \\
\mathscr{C}=\left\{\left(X_{t}\right): \int_{(\tau<\infty)}\left\|X_{\tau}\right\|<\infty, \tau \in T_{c}\right\},
\end{gathered}
$$

$$
\overline{\mathscr{C}}=\left\{\left(X_{t}\right): \text { there is } \sigma \in T_{f} \text { such that } \int_{(\tau<\infty)}\left\|X_{\tau}\right\|<\infty, \sigma \leq \tau \in T_{c}\right\},
$$

$\mathscr{S}=\left\{\left(X_{t}\right):\left(X_{t}, \tau \in T\right)\right.$ converges stochastically (i.e. in probability) in the norm topology $\}$

$$
\mathscr{E}=\left\{\left(X_{t}\right):\left(X_{\tau}, \tau \in T\right) \text { converges essentially in the norm topology }\right\} .
$$

Clearly, $\overline{\mathscr{C}} \supset \mathscr{C} \supset \mathscr{B}$ and $\mathscr{E} \subset \mathscr{S}$. If $\left(\mathscr{F}_{t}\right)$ satisfies the Vitali condition V, particularly, if $D=\mathbf{N} \equiv\{1,2, \ldots\}$, then $\left(X_{t}\right) \in \mathscr{E}$ if and only if $\left(X_{t}\right) \in \mathscr{S}$ (cf. [18], [23], and [20]). Hence, in this case, $\mathscr{S}=\mathscr{E}$.

Mucci ([21], [22]) and Millet and Sucheston ([19], [20]) introduced the notations of martingales in the limit, pramarts, and subpramarts, generalizing those of martingales, amarts (Edgar and Sucheston [7]), uniform amarts (Bellow [2]), and submartingales, and provided some sufficient conditions to ensure that $\left(X_{t}\right) \in \mathscr{S}$ (cf. monographs [10] and [15]). limit if

DefinITION $1([21],[20])$. A stochastic process $\left(X_{t}, \mathscr{F}_{t}, D\right)$ is called a martingale in the

$$
\text { ess } \lim _{t \in D} \operatorname{ess} \sup _{t \leq s \in D}\left\|X_{t}-E\left(X_{s} \mid \mathscr{F}_{t}\right)\right\|=0 \text { a.s. }
$$

† Research supported by the National Natural Science Foundation of China.

Glasgow Math. J. 35 (1993) 239-251. 
Definition $2([\mathbf{1 9}],[\mathbf{2 0}])$. (i) A stochastic process $\left(X_{t}, \mathscr{F}_{t}, D\right)$ is called a pramart if

$$
\text { s. } \lim _{\sigma \leq t ; \sigma, \tau \in T}\left\|X_{\sigma}-E\left(X_{\tau} \mid \mathscr{F}_{\sigma}\right)\right\|=0,
$$

i.e., for each $\epsilon>0$, there exists $\sigma_{0} \in T$ such that, for all $\sigma, \tau \in T$ and $\sigma_{0} \leq \sigma \leq \tau$,

$$
P\left\{\left\|X_{\sigma}-E\left(X_{\tau} \mid \mathscr{F}_{\sigma}\right)\right\|>\epsilon\right\}<\epsilon .
$$
and if

(ii) A stochastic process $\left(X_{t}, \mathscr{F}_{t}, D\right)$ is called a subpramart, if $F$ is a Banach lattice,

$$
\text { s. } \lim _{\sigma \leq \tau ; \sigma, \tau \in T}\left\|\left(X_{\sigma}-E\left(X_{\tau} \mid \mathscr{F}_{\sigma}\right)\right)^{+}\right\|=0 .
$$

Millet and Sucheston [20] proved that if the Vitali condition $\mathrm{V}$ holds (it is also necessary), then every pramart $\left(X_{t}\right)$ is a martingale in the limit. There is a more general class of adapted processes.

Definition 3 [31]. (i) A stochastic process $\left(X_{t}, \mathscr{F}_{t}, D\right)$ is called a mil if

$$
\text { s. } \lim _{\sigma \leq t ; \sigma \in T ; t \in D}\left\|X_{\sigma}-E\left(X_{t} \mid \mathscr{F}_{\mathrm{o}}\right)\right\|=0,
$$

i.e., for each $\epsilon>0$, there exists $\sigma_{0} \in T$ such that, for all $\sigma_{0} \leq \sigma \in T$ and $\sigma \leq t \in D$,

$$
P\left\{\left\|X_{\sigma}-E\left(X_{t} \mid \mathscr{F}_{\sigma}\right)\right\|>\epsilon\right\}<\epsilon .
$$

The mil defined here was called mil(3) in [31], and when $D=\mathbf{N}$, it is equivalent to Talagrand's mil [28] (see [31]). Clearly, pramart $\Rightarrow$ mil, and martingale in the limit $\Rightarrow$ mil [31]. The following theorem was proved by Millet and Sucheston [19].

THEOREM A [19]. Let $\left(X_{n}\right) \equiv\left(X_{n}, n \in \mathbf{N}\right)$ be a pramart of class $\mathscr{B}$. If $F$ has the Radon-Nikodym property, then $\left(X_{n}\right) \in \mathscr{E}$.

In a real-valued case Millet and Sucheston [20] proved this result.

Theorem B [18]. Let $\left(X_{t}\right)$ be a real-valued subpramart satisfying condition (d), i.e.

$$
\lim \inf _{D} \int X_{t}^{-}+\lim \inf _{D} \int X_{t}^{+}<\infty .
$$

Then $\left(X_{t}\right) \in \mathscr{S}$.

Later, Egghe [9], [10], Słaby [26], [27] and Frangos [11] worked on a problem raised by Sucheston: if $F$ has the Radon-Nikodym property, does every $L^{1}$-bounded pramart $\left(X_{t}\right)$ belong to $\mathscr{S}$ ? When $F$ is a Banach lattice, Staby and Frangos proved the following positive subpramart convergence theorem, extending Heinich's positive submartingale convergence theorem [16].

TheOREM C ([11] and [27]). Let $F$ be a Banach lattice with the Radon-Nikodym property. If $\left(X_{t}\right)$ is a positive subpramart satisfying lim inf $\int\left\|X_{t}\right\|<\infty$, and if $\left(\mathscr{F}_{t}\right)$ satisfies the Vitali condition $V$ and $F$ is separable, then $\left(X_{t}\right) \in \mathscr{E}$. 
They also solved Sucheston's problem when $F$ is a separable dual (Frangos [11]), or a weakly sequentially complete space (Staby [27]). The following theorem completely solved the problem.

TheOrem D. (i) ([28], also see [31].) Let $\left(X_{\imath}\right)$ be a mil satisfying lim inf $\int\|X\|<,\infty$. If $F$ has the Radon-Nikodym property, then $\left(X_{t}\right) \in \mathscr{S}$.

(ii) ([31].) If $F=R_{1}$ and $\left(X_{t}\right)$ is a mil satisfying

$$
\liminf \left\{\min \int X_{t}^{+}, \int X_{t}^{-}\right\}<\infty
$$

then $\left(X_{t}\right) \in \mathscr{I}$.

Part (ii) of Theorem $\mathrm{D}$ is an improvement of Mucci's $L^{1}$-bounded, real-valued martingale in the limit convergence theorem [22].

Suggested by Chow's submartingale convergence theorem ([4], also see Remark 2), Yamasaki [34] proved the following theorem.

Theorem E [34]. Suppose that $\left(X_{n}\right)$ is a real-valued martingale in the limit and $\left(X_{n}\right) \in \mathscr{C}$. Then $\left(X_{n}\right) \in \mathscr{E}$.

Yamasaki [34] also provided an example, showing that there exists a real-valued martingale in the limit which belongs to $\mathscr{C}$, but for which $\int X_{n}^{+} \uparrow \infty$.

In this paper we show that Theorem $\mathrm{E}$ can be extended to vector-valued mils, and for pramarts the condition liminf $\int\left\|X_{t}\right\|<\infty$ in Theorem $\mathrm{D}$ can be weakened to $\lim \inf _{\tau \in T} \int\left\|X_{\tau}\right\|<\infty$. On the other hand, it is of interest to characterize a subclass of $\mathscr{S}$. Using Bellow's uniform amart convergence theorem, we can get: if $F$ has the Radon-Nikodym property and the net $\left(X_{\tau}, \tau \in T\right)$ is uniformly integrable, then $\left(X_{n}\right)$ is a uniform amart if and only if $\left(X_{n}\right) \in \mathscr{E}$ (Gut [14]). Krengel and Sucheston [17] also provided an example showing that there exists a real-valued $\left(X_{n}\right) \in \mathscr{B} \cap \mathscr{E}$ such that $\left(X_{n}\right)$ is uniformly integrable, but $\left(X_{n}\right)$ is not an amart. However, Talagrand [28] and Wang and Xue [31] proved that if $\left(X_{t}\right)$ is uniformly integrable, then $\left(X_{t}\right) \in \mathscr{S}$ if and only if $\left(X_{t}\right)$ is a mil, and Xue [32] proved that the converse of Theorem $\mathrm{A}$ is true: if $\left(X_{n}\right) \in \mathscr{S} \cap \mathscr{B}$, then $\left(X_{n}\right)$ is a pramart. In this paper we prove that the converse of Theorem $\mathrm{E}$ is also true. More specifically, we have Theorem 1.

TheOREM 1. Suppose that $F$ has the Radon-Nikodym property.

(a) If $\left(X_{t}\right) \in \overline{\mathscr{C}}$, then

(i) $\left(X_{t}\right) \in \mathscr{S} \Leftrightarrow\left(X_{t}\right)$ is a pramart $\Leftrightarrow\left(X_{t}\right)$ is a mil, and if the Vitali condition $V$ holds,

$$
\left(X_{t}\right) \in \mathscr{S} \Leftrightarrow\left(X_{t}\right) \text { is a martingale in the limit; }
$$

(ii) if $\left(X_{t}\right)$ is a pramart (mil), so is $\left(\left\|X_{t}\right\|\right)$, and under the Vitali condition $V$, if $\left(X_{t}\right)$ is a martingale in the limit, so is $\left(\left\|X_{t}\right\|\right)$.

(b) If $\left(X_{t}\right)$ is a pramart satisfying $\lim \inf _{\tau \in T} \int\left\|X_{\tau}\right\|<\infty$, then $\left(X_{t}\right) \in \mathscr{S}$.

Suppose that $F$ is a Banach lattice. Then $\left(X_{t}\right) \in \mathscr{S} \Rightarrow\left(\left|X_{t}\right|\right) \in \mathscr{S}$, where 
$\left|X_{t}\right|=X_{t}^{+}+X_{t}^{-}$. Since, for $x \in F$,

$$
X_{t} \vee x=\frac{X_{t}+x+\left|X_{t}-x\right|}{2}, \quad X_{t} \wedge x=\frac{X_{t}+x-\left|X_{t}-x\right|}{2}
$$

(cf. [25, Proposition 2.5]), we have the following corollary.

COROLlaRy 1. Suppose that $F$ is a Banach lattice with the Radon-Nikodym property. Then the set of pramarts (mils) of class $\overline{\mathscr{C}}$ is a vector lattice; and, under the Vitali condition $V$, the set of martingales in the limit of class $\overline{\mathscr{C}}$ is also a vector lattice.

When $F$ is a Banach lattice, we have similar results for subpramarts. The part (i) of the following theorem is an improvement of Theorem $\mathrm{C}$.

THEOREM 2. Let $F$ be a Banach lattice with the Radon-Nikodym property and $\left(X_{t}\right)$ a positive subpramart. If one of the following holds, then $\left(X_{t}\right) \in \mathscr{S}$ :

(i) $\lim \inf _{\tau \in T} \int\left\|X_{\tau}\right\|<\infty$;

(ii) $\left(X_{t}\right) \in \overline{\mathscr{C}}$ and $\mathscr{F}_{\infty}$ is nonatomic or s. $\lim \inf _{\tau \in T}\left\|X_{\tau}\right\|<\infty$ a.s., where

$$
\text { s. lim inf }\left\|X_{\tau}\right\|=\operatorname{ess} \sup \left\{\xi: \lim _{\tau \in T} P\left(\left\|X_{\tau}\right\|<\xi\right)=0\right\},
$$

the stochastic lower limit of $\left(\left\|X_{\tau}\right\|, \tau \in T\right)$.

For real-valued processes, we show that condition (d) in Theorem B can be weakened to a one-sided condition and the requirement of being positive in Theorem 2 can be dropped.

THEOREM 3. Let $F=R_{1}$.

(a) If (i) or (ii) holds, then $\left(X_{t}\right) \in \mathscr{S}$ :

(i) $\left(X_{t}\right)$ is a subpramart satisfying $\lim \inf _{\tau \in T} \int X_{\tau}^{+}<\infty$;

(ii) $\left(X_{t}\right)$ is a subpramart or a mil, $\left(X_{t}^{+}\right) \in \overline{\mathscr{C}}$, and $\mathscr{F}_{\infty}$ is nonatomic or s. $\lim \inf _{\tau \in T}\left\|X_{\tau}\right\|<\infty$ a.s. .

(b) If $\left(X_{t}\right) \in \mathscr{S}$ and $\left(X_{t}^{-}\right) \in \overline{\mathscr{C}}$, then $\left(X_{t}\right)$ is a subpramart.

Part of Theorem 3 was proved by Wang [29]. The proof here is new.

Corollary 2. Suppose that $F=R_{1}$. subpramart.

(a) If $\left(X_{t}^{-}\right) \in \overline{\mathscr{C}}$ and $\lim \inf _{\tau \in T} \int X_{\tau}^{+}<\infty$, then $\left(X_{t}\right) \in \mathscr{S}$ if and only if $\left(X_{t}\right)$ is a

(b) If $\left(X_{t}\right) \in \overline{\mathscr{C}}$ and if $\mathrm{s} . \lim \inf _{\tau \in T}\left\|X_{\tau}\right\|<\infty$ a.s. or $\mathscr{F}_{\infty}$ is nonatomic, then

$$
\left(X_{t}\right) \in \mathscr{S} \Leftrightarrow\left(X_{t}\right) \text { is a subpramart } \Leftrightarrow\left(X_{t}\right) \text { is a pramart. }
$$

Austin, Edgar, and Ionescu Tulcea [1] (also see [7]) proved that $L^{1}$-bounded, real-valued amarts form a vector lattice. This result was extended by Ghoussoub [12] to $L^{\prime}$-bounded, Banach lattice-valued order amarts when the Radon-Nikodym property holds, and by Schmidt [24] to $L^{1}$-bounded, $l^{1}$-valued uniform amarts. It is natural to ask: can we change "class $\overline{\mathscr{C}}$ " to " $L$-bounded class" in Corollary 1 ? For martingales in the 
limit and mils, it is impossible. Indeed, Bellow and Dvoretzky [3] presented an example showing that there exists a uniformly integrable, real-valued martingale in the limit $\left(X_{n}\right)$ such that $\left(\left|X_{n}\right|\right)$ is not a martingale in the limit, and Talagrand [28] constructed an $L^{1}$-bounded, real-valued martingale in the limit $\left(X_{n}\right)$ with $X_{n} \rightarrow 0$ a.s. such that $\left(\left|X_{n}\right|\right)$ is not a mil. Hence, the set of $L^{1}$-bounded, real-valued martingales in the limit (mils) is not a vector lattice. Talagrand also presented an $L^{1}$-bounded, $l^{2}$-valued pramart $\left(X_{n}\right)$ such that $\left(\left|X_{n}\right|\right)$ is not a pramart. However, Talagrand [28] and Wang [29] proved that the set of $L^{1}$-bounded, real-valued pramarts is a vector lattice. In fact, Talagrand and Wang proved that if $\left(X_{n}\right)$ is a real-valued pramart satisfying $\lim \inf \int\left|X_{n}\right|<\infty$, then so is $\left(\left|X_{n}\right|\right)$. The following theorem is an improvement of their result.

THEOREM 4. If $\left(X_{t}\right)$ is a real-valued pramart satisfying $\lim \inf _{\tau \in T} \min \left\{\int X_{\tau}^{-}, \int X_{\tau}^{+}\right\}<\infty$, then $\left(\left|X_{t}\right|\right)$ is a pramart, hence, for each $\lambda \in R_{1}$, both $\left(X_{t} \vee \lambda\right)$ and $\left(X_{t} \wedge \lambda\right)$ are pramarts.

In Section 2 we characterize real-valued pramarts and subpramarts via Snell's envelopes. We prove Theorems 1-4 in Section 3. In Section 4 we make some comments pointing out that: (i), for pramarts, the condition $\lim \inf _{\tau \in T} \int\left\|X_{\tau}\right\|<\infty$ is weaker than the condition liminf $\int\left\|X_{t}\right\|<\infty$; (ii), in general, the condition $\left(X_{t}^{-}\right) \in \overline{\mathscr{C}}$ in Theorem 3 cannot be dropped; (iii) the condition $\lim \inf _{\tau \in T} \min \left\{\int X_{\tau}^{+}, \int X_{\tau}^{-}\right\}<\infty$ in Theorem 4 is necessary; (iv), in general, the set $\overline{\mathscr{C}}$ is larger than $\mathscr{C}$.

2. Snell's envelopes and characterizations of pramarts and subpramarts. In this section we assume that $F=R_{1}$. We use the following Snell's envelopes to characterize real-valued pramarts and subpramarts. For a real-valued process $\left(X_{i}\right)$ we denote

$$
Y_{t}=\operatorname{ess} \sup _{t \leq \tau \in T} E\left(X_{\tau} \mid \mathscr{F}_{t}\right), \quad P_{t}=\operatorname{ess} \sup _{t \leq \tau \in T} E\left(X_{\tau}^{+} \mid \mathscr{F}_{t}\right), \quad R_{t}=\operatorname{ess} \inf _{t \leq \tau \in T} E\left(X_{\tau} \mid \mathscr{F}_{t}\right) .
$$

The following lemma is well known in the martingale theory and the theory of optimal stopping (cf. [5], [13], and [23]).

LEMMA 1. $\left(Y_{\tau}, \mathscr{F}_{\tau}, T\right)$ is a generalized supermartingale (i.e. $Y_{\tau}$ takes values in $(-\infty, \infty], E Y_{\tau}^{-}<\infty$ and $E\left(Y_{\tau} \mid \mathscr{F}_{\sigma}\right) \leq Y_{\sigma}$ a.s. for all $\tau, \sigma \in T$ and $\left.\tau \geq \sigma\right)$, and for any $\tau \in T$ there exist $\left(\tau_{n}\right) \subset T$ such that $\tau \leq \tau_{n}$ and

$$
E\left(X_{\tau_{n}} \mid \mathscr{F}_{\tau}\right) \uparrow Y_{\tau}=\operatorname{ess} \sup \left\{E\left(X_{\sigma} \mid \mathscr{F}_{\tau}\right): \tau \leq \sigma \in T\right\} .
$$

Therefore, if $\int\left|Y_{t}\right|<\infty, t \in D$, then $\left(-Y_{t}\right)$ is a subpramart. Moreover, if $\sup _{t} \int\left|Y_{t}\right|<\infty$, then $\left(Y_{t}\right)$ is an amart, hence a pramart.

Proposition 1. (i) $\left(X_{t}\right)$ is a subpramart if and only if $\left(X_{\tau}-R_{\tau}, \tau \in T\right)$ converges to zero in probability. In this case, $\lim _{\tau \in T} P\left(R_{\tau}=-\infty\right)=0$.

(ii) $\left(X_{t}\right)$ is a pramart if and only if $\left(Y_{\tau}-R_{\tau}, \tau \in T\right)$ converges to zero in probability. In this case, $\lim _{\tau \in T} P\left(R_{\tau}=-\infty\right)=\lim _{\tau \in T} P\left(Y_{\tau}=\infty\right)=0$. 
Proof. For any $\tau \in T$, by Lemma 1 , we can choose $\tau \leq \tau_{n} \in T$ such that $E\left(X_{\tau_{n}} \mid \mathscr{F}_{\tau}\right) \downarrow R_{\tau}$. Since $X_{t} \geq R_{t}$ a.s.,

$$
\begin{aligned}
\sup _{\tau \leq \sigma \in T} P\left(X_{\tau}-E\left(X_{\sigma} \mid \mathscr{F}_{\tau}\right)>\epsilon\right) & \leq P\left(\text { ess } \sup _{\tau \leq \sigma \in T}\left(X_{\tau}-E\left(X_{\sigma} \mid \mathscr{F}_{\tau}\right)\right)>\epsilon\right) \\
=P\left(X_{\tau}-R_{\tau}>\epsilon\right) & =P\left(\left|X_{\tau}-R_{\tau}\right|>\epsilon\right)=\int \lim _{n} I\left(X_{\tau}-E\left(X_{\tau_{n}} \mid \mathscr{F}_{\tau}\right)>\epsilon\right) \\
& =\lim _{n} \int I\left(X_{\tau}-E\left(X_{\tau_{n}} \mid \mathscr{F}_{\tau}\right)>\epsilon\right) \leq \sup _{\tau \leq \sigma \epsilon T} P\left(X_{\tau}-E\left(X_{\sigma} \mid \mathscr{F}_{\tau}\right)>\epsilon\right) .
\end{aligned}
$$

Hence, $\left(X_{t}\right)$ is a subpramart if and only if $\left(X_{\tau}-R_{\tau}, \tau \in T\right)$ converges to zero in probability. In this case, $\lim _{\tau \in T} P\left(R_{\tau}=-\infty\right) \leq \lim _{\tau \in T} P\left(X_{\tau}-R_{\tau}>1\right)=0$. Finally (ii) follows from (i) and the symmetric property.

COROllary 3. Suppose that $\left(X_{\tau}, \tau \in T\right)$ converges to zero in probability. Then

(i) $\left(X_{t}\right)$ is a subpramart if and only if $\left(R_{\tau}, \tau \in T\right)$ converges to zero in probability;

(ii) if, in addition, $\left(X_{t}\right)$ is nonnegative, then $\left(X_{t}\right)$ is a pramart if and only if $\left(Y_{\mathfrak{r}}, \tau \in T\right)$ converges to zero in probability.

Remark 1. When $\left(X_{t}\right)$ is nonpositive, (i) in Proposition 1 was proved by Millet and Sucheston [20]. The proof here is adopted from their paper. When $D=\mathbf{N}$, (ii) in Corollary 3 is an analogue of Theorem 11 in [28].

3. Proofs of Theorems 1-4. To prove Theorems 1-4, we need the following lemmas. Let

$$
W_{t}=\operatorname{ess} \sup _{t \leq \tau \in T} E\left(\left\|X_{\tau}\right\| \mid \mathscr{F}_{t}\right), \quad A_{t}=\left(W_{t}=\infty\right)
$$

Definition 4 (cf. $[\mathbf{1 0}])$. Let $F$ be a Banach lattice. A stochastic process $\left(X_{t}, \mathscr{F}_{t}, D\right)$ is called a GBT (a game which becomes better with time), if

$$
\text { s. } \lim _{t \leq s ; t, s \in D}\left\|\left(X_{t}-E\left(X_{s} \mid \mathscr{F}_{t}\right)\right)^{+}\right\|=0 .
$$

Lemma 2. (i) ([33]) $A_{t} \subset A_{s}$ a.s. for all $s \leq t$. Hence $A^{*} \equiv \operatorname{ess} \lim A_{1}$ exists.

(ii) If $\left(X_{t}\right) \in \overline{\mathscr{C}}$, then $P\left(A^{*}\right)=0$ or $A^{*}$ is a union of atoms of $\mathscr{F}_{\infty}$.

Proof. When $\left(X_{t}\right) \in \mathscr{C}$, (ii) was proved in [33]. Now assume that $\left(X_{t}\right) \in \overline{\mathscr{C}}$ and for some $\sigma \in T_{f}$ and each $\sigma \leq \tau \in T_{c}, \int_{(\tau<\infty)}\left\|X_{\tau}\right\|<\infty$. Choose $\left(t_{n}\right) \subset D$ such that $P\left(\sigma<t_{n}\right) \uparrow 1$. Since $\left(X_{t} I\left(\sigma \leq t_{n}\right), \mathscr{F}_{t}, t_{n} \leq t \in D\right) \in \mathscr{C}, A^{*} \cap\left(\sigma \leq t_{n}\right)$ is a union of atoms of $\mathscr{F}_{\infty}$ or $\phi, n \geq 1$, and so is $A^{*}$.

Lemma 3. Suppose that $\left(X_{t}\right)$ is a real-valued GBT (subpramart or mil) and $\left(X_{t}^{+}\right) \in \overline{\mathscr{C}}$. Then $\left(X_{t}\right)\left(\left(X_{\tau}, \tau \in T\right)\right)$ converges stochastically to a r.v. $\xi$ such that $-\infty<\xi \leq+\infty$, $(\xi=+\infty)=A^{+*}$ a.s., and $A^{+*}$ is a union of atoms of $\mathscr{F}_{\infty}$ or $\phi$, where $A^{+*}=\operatorname{ess} \lim A_{t}^{+}$, $A_{t}^{+}=\left(P_{t}=\infty\right)$. 
Proof. As the proof of Lemma 2 we may assume that $\left(X_{t}^{+}\right) \in \mathscr{C}$. Then the conclusions for GBTs and subpramarts follow from Theorems 9 and 10 in [33]. Now assume that $\left(X_{t}\right)$ is a mil. Choose $\left(t_{n}\right) \subset D$ such that $A_{t_{n}}^{+} \downarrow A^{+*}$. For any fixed $n \geq 1$ and $K \geq 1$, by Lemma 1 , it is easy to see that $\left(X_{t} I\left(P_{t_{n}} \leq K\right), t \geq t_{n}\right)$ is a mil and

$$
\lim \inf E X_{t}^{+} I\left(P_{t_{n}} \leq K\right) \leq E P_{t_{n}} I\left(P_{t_{n}} \leq K\right)<\infty .
$$

Hence $\left(X_{t} I\left(P_{t_{n}} \leq K\right)\right) \in \mathscr{S}$ (Theorem D). Since $\bigcup_{n \geq 1} \bigcup_{K \geq 1}\left(P_{t_{n}} \leq K\right)=\Omega \backslash A^{+*}$, $\left(X_{t} I\left(\Omega \backslash A^{+*}\right)\right) \in \mathscr{S}$. Since every mil is a GBT, s $\lim _{T} X_{\tau} I\left(A^{+*}\right)=\infty I\left(A^{+*}\right)$.

Remark 2. Suppose that $\left(X_{n}\right)$ is a real-valued process and $\left(X_{n}^{+}\right) \in \mathscr{C}$. Chow [4] proved that if $\left(X_{n}\right)$ is a submartingale, then $\left(X_{n}\right)$ converges a.s. to a r.v. $\xi$ which takes values in $(-\infty,+\infty]$. Yamasaki [34] obtained the same result for martingales in the limit. In Lemma 3, we extend their results to subpramarts and mils and show that $(\xi=+\infty)$ is a union of atoms of $\mathscr{F}_{\infty}$ or $\phi$.

Let $F^{*}$ be the dual space of $F$ and $F^{*+}$ the positive cone of $F^{*}$ if $F$ is a Banach lattice.

Lemma 4.(i) Suppose that $F$ is a Banach lattice. If $\left(X_{t}\right)$ is a positive $G B T$, then so are $\left(\left\|X_{t}\right\|\right)$ and $\left(f\left(X_{t}\right)\right), f \in F^{*+}$.

(ii) If $F$ is a Banach lattice and $\left(X_{t}\right)$ is a positive subpramart, then so are $\left(\left\|X_{t}\right\|\right)$ and $\left(f\left(X_{t}\right)\right), f \in F^{*+}$.

(iii) If $\left(X_{t}\right)$ is a mil, then $\left(\left\|X_{t}\right\|\right)$ is a $G B T$ and $\left(f\left(X_{t}\right)\right)$ is a mil, $f \in F^{*}$.

Proof. If $F$ is a Banach lattice and $\left(X_{t}\right)$ is positive, then for $t, s \in D$ and $\sigma \in T$,

$$
\left\|\left(X_{t}-E\left(X_{s} \mid \mathscr{F}_{t}\right)\right)^{+}\right\| \geq\left(\left\|X_{t}\right\|-\left\|E\left(X_{s} \mid \mathscr{F}_{t}\right)\right\|\right)^{+} \geq\left(\left\|X_{t}\right\|-E\left(\left\|X_{s}\right\| \mid \mathscr{F}_{t}\right)\right)^{+},
$$

and

$$
\left\|\left(X_{t}-E\left(X_{s} \mid \mathscr{F}_{t}\right)\right)^{+}\right\| \geq \frac{f\left(X_{t}-E\left(X_{s} \mid \mathscr{F}_{t}\right)\right)^{+}}{\|f\|} \geq \frac{\left(f\left(X_{t}\right)-E\left(f\left(X_{s}\right) \mid \mathscr{F}_{t}\right)\right)^{+}}{\|f\|}, f \in F^{*+} \backslash 0,
$$

(i) holds. Similarly, we get (ii). Since

$$
\left\|X_{t}-E\left(X_{s} \mid \mathscr{F}_{t}\right)\right\| \geq\left\|X_{t}\right\|-E\left(\left\|X_{s}\right\| \mathscr{F}_{t}\right),
$$

and

$$
\left\|X_{o}-E\left(X_{s} \mid \mathscr{F}_{\sigma}\right)\right\| \geq \frac{\left|f\left(X_{\sigma}-E\left(X_{s} \mid \mathscr{F}_{\sigma}\right)\right)\right|}{\|f\|}=\frac{\left|f\left(X_{\sigma}\right)-E\left(f\left(X_{s}\right) \mid \mathscr{F}_{\sigma}\right)\right|}{\|f\|}, f \in F^{*} \backslash 0,
$$

(iii) holds.

Lemma 5. Suppose that $\left(X_{t}\right)$ is a mil and $\left(X_{t}\right) \in \overline{\mathscr{C}}$. Then $P\left(A^{*}\right)=0$.

Proof. Assume that $P\left(A^{*}\right)>0$. By Lemma 2, we may assume that $A^{*}$ is an atom of $\mathscr{F}_{\infty}$ and $X_{t}=x_{t}$ on $A^{*}$. Since $\left(X_{t}\right)$ is a mil, by Lemmas 3 and 4 ,

$$
\lim _{t} f\left(x_{t}\right) \text { exists and is finite for each } f \in F^{*},
$$


and

$$
\left\|x_{t}\right\| \rightarrow \infty
$$

which contradicts the Banach-Steinhaus theorem.

When $D=N$, the following lemma was proved in [32]. The proof here is new.

Lemma 6. Assume that $\left(X_{t}\right) \in \mathscr{B} \cap \mathscr{S}$. Then $\left(X_{t}\right)$ is a pramart.

Proof. Assume that $\left(X_{\tau}, \tau \in T\right)$ converges stochastically to $X$. By Fatou's lemma, $X \in L^{1}$. Let

$$
Z_{t}=X_{t}-E\left(X \mid \mathscr{F}_{t}\right) \text {. }
$$

Then $\left(Z_{t}\right) \in \mathscr{B}$. Clearly, we need only to show that $\left(Z_{t}\right)$ is a pramart. Since $\left(E\left(X \mid \mathscr{F}_{\tau}\right)\right.$, $\tau \in T)$ converges stochastically to $X,\left(\left\|Z_{\tau}\right\|, \tau \in T\right)$ converges stochastically to zero. Hence, we need only to show that $\left(S_{\tau}, \tau \in T\right)$ converges stochastically to zero, where

$$
S_{t}=\operatorname{ess} \sup _{t \leq \sigma \in T} E\left(\left\|Z_{\sigma}\right\| \mid \mathscr{F}_{t}\right)
$$

First we show that $\left(S_{t}\right) \in \mathscr{P}$. By Lemma $1,\left(S_{t}\right)$ is a pramart satisfying

$$
\sup _{\imath \in D} \int\left|S_{t}\right|=\sup _{\tau \in T} \int\left\|Z_{\tau}\right\| \leq \sup _{\tau \in T} \int\left\|X_{\tau}\right\|+\int\|X\|<\infty .
$$

Hence, by Theorem B, $\left(S_{t}\right) \in \mathscr{S}$. Assume that $\left(S_{\tau}, \tau \in T\right)$ does not converge stochastically to zero, then $\left(\left\|Z_{\tau}\right\|-S_{\tau}, \tau \in T\right)$ converges stochastically to a nonpositive r.v. $\xi$ such that $P(\xi<0)>0$, since $S_{t} \geq\left\|Z_{t}\right\|$ and $\left(S_{t}\right),\left(\left\|Z_{t}\right\|\right) \in \mathscr{S}$. Then, by Fatou's lemma,

$$
\lim _{\tau \in T} \inf \int\left(S_{\tau}-\left\|Z_{\tau}\right\|\right) \geq \int \lim _{\tau \in T}\left(S_{\tau}-\left\|Z_{\tau}\right\|\right)>0 .
$$

On the other hand, by Lemma 1 ,

$$
\lim _{\tau \in T} \int S_{\tau}=\lim _{\tau \in T}\left(\sup _{\tau \leq \tau^{\prime} \in T} \int\left\|Z_{\tau^{\prime}}\right\|\right)=\lim \sup _{\tau \in T} \int\left\|Z_{\tau}\right\|<\infty,
$$

which contradicts (2).

LEMMA 7. If $\left(X_{t}\right) \in \mathscr{P} \cap \overline{\mathscr{C}}$, then $P\left(A^{*}\right)=0$.

Proof. Assume that $\left(X_{t}\right) \in \mathscr{S} \cap \overline{\mathscr{C}}$ and $P\left(A^{*}\right)>0$. Then, by Lemma 2 , we may assume that $A^{*}$ is an atom of $\mathscr{F}_{\infty}$, and $A_{t}$ is an atom of $\mathscr{F}_{t}$ such that $A_{t} \supset A^{*},\left\|X_{t}\right\|=a_{t}$ on $A_{t}$, and $\lim a_{t}=a \in R_{1}$. We may also assume that $a_{t}<a+1, t \in D$. For any $\sigma \in T_{f}$, there is $t_{0} \in D, P\left(A^{*} \cap\left(\sigma=t_{0}\right)\right)>0$. Since $\left(\sigma=t_{0}\right) \in \mathscr{F}_{t_{0}}, A_{t_{0}} \subset\left(\sigma=t_{0}\right)$. Since $W_{t_{0}}=\infty$ on $A_{t_{0}}$, there is $t_{0} \leq \tau_{1} \in T$ such that $E\left(\left\|X_{\tau_{1}}\right\| \mathscr{F}_{t_{0}}\right)>(a+2) / P\left(A_{t_{0}}\right)$ on $A_{t_{0}}$, and there is $t_{1} \in D$ such that $P\left(A^{*} \cap\left(\tau_{1}=t_{1}\right)\right)>0$ (then $A_{t_{1}} \subset\left(\tau_{1}=t_{1}\right)$ ). Assume that we have chosen $t_{n-1} \leq \tau_{n} \in T$ such that $E\left(\left\|X_{t_{n}}\right\| \mid \mathscr{F}_{t_{n-1}}\right) \geq(a+2) / P\left(A_{t_{n-1}}\right)$ on $A_{t_{n-1}}$. Then there is $t_{n} \in D$ such that $P\left(A^{*} \cap\right.$ $\left.\left(\tau_{n}=t_{n}\right)\right)>0$, (hence $A_{t_{n}} \subset\left(\tau_{n}=t_{n}\right)$ ). And we can choose $t_{n} \leq \tau_{n+1} \in T$ such that $E\left(\left\|X_{\tau_{n+1}}\right\| \mid \mathscr{F}_{t_{n}}\right) \geq(a+2) / P\left(A_{t_{n}}\right)$ on $A_{t_{n}}$. Let $\tau=\sum_{n \geq 1} \tau_{n} I\left(A_{t_{n-1}} \backslash A_{t_{n}}\right)+\sigma I\left(\Omega \backslash A_{t_{0}}\right)+\infty I\left(A^{*}\right)$. 
Then $\sigma \leq \tau \in T_{c}$ and

$$
\begin{aligned}
E\left\|X_{\tau}\right\| I(\tau<\infty) & \geq \sum_{n}\left(E\left\|X_{\tau_{n}}\right\| I\left(A_{t_{n-1}}\right)-E\left\|X_{t_{n}}\right\| I\left(A_{t_{n}}\right)\right) \\
& \geq \sum_{n}\left(E\left\|X_{\tau_{n}}\right\| I\left(A_{t_{n-1}}\right)-(a+1)\right) \geq \sum_{n}\left(E\left(E\left(\left\|X_{\tau_{n}}\right\| \mathscr{F}_{t_{n-1}}\right) I\left(A_{t_{n-1}}\right)-(a+1)\right)=\infty .\right.
\end{aligned}
$$

Hence, for any $\sigma \in T_{f}$ there is $\sigma \leq \tau \in T_{c}$ such that $E\left\|X_{\tau}\right\| I(\tau<\infty)=\infty$, i.e. $\left(X_{t}\right) \notin \overline{\mathscr{C}}$, a contradiction.

Proof of Theorem 1. (a) Since $\left(X_{t}\right) \in \overline{\mathscr{C}}$, by Lemmas 5 and 7 , either $\left(X_{t}\right) \in \mathscr{S}$ or being a mil implies $P\left(A^{*}\right)=0$, and we can choose $\left(t_{k}\right) \subset D$ such that $t_{1}<t_{2}<\ldots$ and $P\left(A_{t_{k}}\right) \downarrow 0$. For $m, k \in \mathbf{N}$ define

$$
X_{t}^{k}=X_{t} I\left(W_{t_{k}}<m\right), \quad t_{k} \leq t \in D .
$$

Since $E\left(\left\|X_{\tau}\right\| \mathscr{F}_{t}\right) \leq W_{t}$, a.s., $t \leq \tau \in T$, by Lemma 1 ,

$$
\sup _{t_{k} \leq \tau \in T} \int\left\|X_{\tau}^{k}\right\| \leq \int W_{t_{k}}\left(W_{t_{k}}<m\right)<\infty .
$$

Hence, $\left(X_{t}^{k}, t_{k} \leq t \in D\right)$ is of class $\mathscr{B}$. If $\left(X_{t}\right)$ is a mil, then $\left(X_{t}^{k}, t_{k} \leq t \in D\right)$ is a mil of class $\mathscr{B}$, and by Theorem $\mathrm{D},\left(X_{t}^{k}, t_{k} \leq t \in D\right) \in \mathscr{P}$; if $\left(X_{t}\right) \in \mathscr{P}$, then, $\left(X_{t}^{k}, t_{k} \leq t \in D\right) \in \mathscr{B} \cap \mathscr{S}$, and, by Lemma $6,\left(X_{t}^{k}, t_{k} \leq t \in D\right)$ is a pramart. Since $\bigcup_{k, m \in N}\left(W_{t_{k}}<m\right)=\Omega \backslash A^{*}=\Omega$ a.s. and every pramart is a mil (a martingale in the limit, if the Vitali condition holds), we get (a).

Now we prove (b). Assume that $\left(X_{t}\right)$ is a pramart satisfying $\lim \inf _{\tau \in T} \int\left\|X_{\tau}\right\|<\infty$. It is easy to show that $\left(X_{\tau}, \mathscr{F}_{\tau}, \tau \in T\right)$ is a mil, and applying Theorem $\mathrm{D},\left(X_{t}\right) \in \mathscr{S}$.

Proof of Theorem 2. Proof of (i). Suppose that $F$ has the Radon-Nikodym property and $\left(X_{t}\right)$ is a positive subpramart satisfying $\lim \inf _{\tau \in T} \int\left\|X_{\tau}\right\|=M<\infty$. Choose $\left(t_{n}\right) \subset D$ such that $t_{1} \leq t_{2} \leq \ldots$ and

$$
\sup _{t_{n} \leq \tau \leq \sigma ; \tau, \sigma \in T} P\left(\left\|\left(X_{\tau}-E\left(X_{\sigma} \mid \mathscr{F}_{\tau}\right)\right)^{+}\right\|>1 / n\right)<2^{-n} .
$$

We claim that the following fact holds:

$$
\begin{aligned}
& \text { for all }\left(\tau_{n}, \sigma_{n}\right) \subset T \text { such that } t_{n} \leq \tau_{n} \leq \sigma_{n} \leq \tau_{n+1}, \text { and } \int\left\|X_{\sigma_{n}}\right\|<M+1, \\
& \left(X_{\tau_{1}}, X_{\sigma_{1}}, \ldots, X_{\tau_{n}}, X_{\sigma_{n}}, \ldots\right) \text { converges almost surely to a finite r.v. }
\end{aligned}
$$

Proof of the claim. For $n \geq 1$, let

$$
\begin{aligned}
\bar{X}_{2 n-1}=X_{\tau_{n}}, & \overline{\mathscr{F}}_{2 n-1}=\mathscr{F}_{\tau_{n}} ; \\
\bar{X}_{2 n}=X_{\sigma_{n}}, & \overline{\mathscr{F}}_{2 n}=\mathscr{F}_{\sigma_{n}} .
\end{aligned}
$$

Then, $\left(\bar{X}_{n}, \overline{\mathscr{F}}_{n}, n \in \mathbf{N}\right)$ is a positive subpramart satisfying

$$
\liminf \int\left\|\bar{X}_{n}\right\| \leq \liminf \int\left\|X_{\sigma_{n}}\right\|<\infty .
$$


Since $\bar{X}_{n}$ being Bochner integrable is separably valued, so is $\left(\bar{X}_{n}, n \in \mathbf{N}\right)$. By Theorem C, $\left(\bar{X}_{n}, n \in \mathbf{N}\right) \in \mathscr{E}$, and the claim has been proved.

The above claim implies that $\left(X_{t}\right) \in \mathscr{S}$. In fact, if $\left(X_{t}\right) \notin \mathscr{S}$, then there is a $c>0$ such that for any $t \in D$ there exist $t \leq \tau, \rho \in T$,

$$
P\left(\left\|X_{\tau}-X_{\rho}\right\|>c\right)>c .
$$

Choose $t_{1} \leq \tau_{1}, \rho_{1} \in T$ such that (4) holds. Pick $\sigma_{1} \in T$ such that $\sigma_{1} \geq \tau_{1}, \sigma_{1} \geq \rho_{1}, \sigma_{1} \geq t_{2}$, and $\int\left\|X_{\sigma_{1}}\right\|<M+1$. Assume that $\tau_{n}, \rho_{n}$ and $\sigma_{n}$ have been chosen, choose $\tau_{n+1}, \rho_{n+1}$ and $\sigma_{n+1} \in T$ such that $\tau_{n+1} \geq \sigma_{n}, \rho_{n+1} \geq \sigma_{n}$, and $\sigma_{n+1} \geq \tau_{n+1}, \sigma_{n+1} \geq \rho_{n+1}, \sigma_{n+1} \geq t_{n+2}$, $\int\left\|X_{o_{n+1}}\right\|<M+1$, and

$$
P\left(\left\|X_{\tau_{n+1}}-X_{\rho_{n+1}}\right\|>c\right)>c .
$$

Then, by the claim,

$$
\left(X_{\tau_{1}}, X_{\sigma_{1}}, \ldots, X_{\tau_{n}}, X_{\sigma_{n}}, \ldots\right)
$$

and

$$
\left(X_{\rho_{1}}, X_{\sigma_{1}}, \ldots, X_{\rho_{n}}, X_{\sigma_{n}}, \ldots\right)
$$

converge almost surely to finite r.v.s. Hence,

$$
\left(X_{\tau_{1}}, X_{\rho_{1}}, \ldots, X_{\tau_{n}}, X_{\rho_{n}}, \ldots\right)
$$

converges almost surely to a finite r.v., which contradicts (5).

Proof of (ii). Under the assumptions of (ii), by Lemmas 2 and 3, $P\left(A^{*}\right)=0$. Define $X_{t}^{k}$ as that in the proof of Theorem 1 . Then $\left(X_{t}^{k}, t_{k} \leq t \in D\right)$ is a positive, $L^{1}$-bounded subpramart, therefore, by part (i) of Theorem $2,\left(X_{t}^{k}, t_{k} \leq t \in D\right) \in \mathscr{S}$, which implies $\left(X_{t}\right) \in \mathscr{S}$.

Proof of Theorem 3. (a) Assume that $\left(X_{t}\right)$ is a real-valued subpramart and $\lim \inf _{\tau \in T} \int X_{\tau}^{+}<\infty$. By Proposition 1 , we can choose $\left(t_{n}\right) \subset D$ such that $t_{1} \leq t_{2} \leq \ldots$ and $P\left(R_{t_{n}}=-\infty\right) \rightarrow 0$. For $M>0$, and $t_{n} \leq t \in D$, by Lemma 1 ,

$$
\begin{aligned}
\int\left|R_{t}\right| I\left(R_{t_{n}}>-M\right) & =\int-R_{t} I\left(R_{t_{n}}>-M\right)+2 \int R_{t}^{+} I\left(R_{t_{n}}>-M\right) \\
& \leq \int-R_{t_{n}} I\left(R_{t_{n}}>-M\right)+2 \sup _{s \in D} \int R_{s}^{+} \\
& \leq M+2 \sup _{s \in D}\left(\inf _{s \leq \tau \in T} \int X_{\tau}^{+}\right)=M+2 \lim _{\tau \in T} \inf _{\tau \in T} \int X_{\tau}^{+}<\infty .
\end{aligned}
$$

Hence, $\left(R_{t} I\left(R_{t_{n}}>-M\right), t_{n} \leq t \in D\right)$ is an $L^{1}$-bounded pramart, and $\left(R_{t} I\left(R_{t_{n}}>-M\right)\right) \in \mathscr{P}$ (Theorem B). By Proposition 1, $\left(X_{t} I\left(R_{t_{n}}>-M\right)\right) \in \mathscr{S}$. Since $\cup_{n}\left(R_{t_{n}}>-\infty\right)=\Omega$ a.s., $\left(X_{t}\right) \in \mathscr{S}$. The proof of (ii) is similar to the proof in Theorem 2 and, therefore, is omitted.

(b) Now we assume that $\left(X_{\imath}^{-}\right) \in \overline{\mathscr{C}} \cap \mathscr{\mathscr { S }}$. For any $\epsilon>0$, by Theorem 1 , we can choose $M>0$ and $\rho \in T$ such that

$$
\sup _{\rho \leq \tau \in T} P\left(X_{\tau}>M\right)<\epsilon
$$


and

$$
\sup _{\rho \leq \tau \leq \sigma ; \tau, \sigma \in T} P\left(X_{\tau} \wedge M-E\left(X_{\sigma} \wedge M \mid \mathscr{F}_{\tau}\right)>\epsilon\right)<\epsilon,
$$

since $(X, \wedge M) \in \overline{\mathscr{C}} \cap \mathscr{S}$ and it is a pramart (Theorem 1). Hence, for any $\tau, \sigma \in T$ and $\rho \leq \tau \leq \sigma$,

$$
\begin{aligned}
P\left(X_{\tau}-E\left(X_{\sigma} \mid \mathscr{F}_{\tau}\right)>\epsilon\right) & \leq P\left\{\left(X_{\tau}-E\left(X_{\sigma} \wedge M \mid \mathscr{F}_{\tau}\right)\right) I\left(X_{\tau} \leq M\right)>\epsilon\right\}+P\left(X_{\tau}>M\right) \\
& \leq P\left(X_{\tau} \wedge M-E\left(X_{\sigma} \wedge M \mid \mathscr{F}_{\tau}\right)>\epsilon\right)+\epsilon<2 \epsilon,
\end{aligned}
$$

i.e. $\left(X_{t}\right)$ is a subpramart.

Proof of Theorem 4. Without loss of generality we may and do assume that $\lim \inf _{\tau \in T} \int X_{\tau}^{-}<\infty$. By Theorem 3, $\left(X_{t}\right) \in \mathscr{S}$. The condition $\lim \inf _{\tau \in T} \int X_{\tau}^{-}<\infty$ implies $\left(P_{t}=\infty\right)=\left(Y_{t}=\infty\right)$ a.s. (see [33, Lemma 3]), hence, by Proposition 1, we can choose $\left(t_{k}\right) \subset D$ such that

$$
P\left(P_{t_{k}}=\infty\right)=P\left(Y_{t_{k}}=\infty\right) \rightarrow 0 \text {. }
$$

Then, it is easy to show that for each $k \geq 1$ and $M>0,\left(X_{t}^{+} I\left(P_{t_{k}}<M\right), t \geq t_{k}\right) \in \overline{\mathscr{C}} \cap \mathscr{S}$. Applying Theorem $1,\left(X_{t}^{+} I\left(P_{t_{k}}<M\right), t \geq t_{k}\right)$ is a pramart, and, by $(6),\left(X_{t}^{+}\right)$is also a pramart. Therefore, $\left(\left|X_{t}\right|\right)=\left(2 X_{t}^{+}-X_{t}\right)$ is a pramart. For each $\lambda \in R_{1}$, by (1), both $(X, \wedge \lambda)$ and $\left(X_{t} \vee \lambda\right)$ are pramarts.

4. Some comments. 1. For a martingale $\left(X_{n}\right)$, clearly,

$$
\lim \inf _{\tau \in T} \int\left\|X_{\tau}^{+}\right\|<\infty \Leftrightarrow \lim \inf _{n} \int\left\|X_{n}^{+}\right\|<\infty .
$$

However, for subpramarts, condition $\lim \inf _{\tau \in T} \int\left\|X_{\tau}^{+}\right\|<\infty$ is weaker than condition $\lim \inf _{n} \int\left\|X_{n}^{+}\right\|<\infty$ : let $\Omega=(0,1], \mathscr{F}=\mathscr{F}_{n}$ be the class of Borel sets of $(0,1]$, and $P$ the Lebesgue measure. Define $X_{n}=n^{2} I(0,1 / n]$. Then $\left(X_{n}\right)$ is a positive pramart, $\int X_{n} \uparrow \infty$, and $\lim \inf _{\tau \in T} \int X_{\tau}=0$.

2. The following example shows that, in general, the condition $\left(X_{t}^{-}\right) \in \overline{\mathscr{C}}$ in Theorem 3 can not be dropped.

Let $\left(X_{n}\right)$ be independent r.v.s such that $X_{n} \leq 0, \int X_{n} \downarrow-\infty$ and $X_{n} \rightarrow 0$ a.s. and $X_{n}$ is non-degenerate. Let $\mathscr{F}_{n}=\sigma\left(X_{1}, \ldots, X_{n}\right)$. Clearly, $\left(X_{n}\right)$ is not a subpramart, and we can show that $\left(X_{n}^{-}\right) \notin \overline{\mathscr{C}}$. In fact, for each $\sigma \in T_{f}$, choose $n \in \mathbf{N}$ so large that $P(\sigma \leq n)>0$. Then we can find $\left(A_{k}, k \geq n\right)$ such that $A_{k} \subset(\sigma \leq n), A_{k} \in \mathscr{F}_{k}, P\left(A_{k}\right)>0, P\left(A_{k}, A_{j}\right)=0$, $j \neq k$. Choose $n_{k} \geq k, E\left|X_{n_{k}}\right| P\left(A_{k}\right)>1$. Let $\tau=\sum_{k \geq n} n_{k} I\left(A_{k}\right)+\infty I\left(\Omega \backslash\left(\bigcup_{k} A_{k}\right)\right.$. Then $\sigma \leq \tau \in T_{c}$ and $\int_{(\tau<\infty)}\left|X_{\tau}\right|=\infty,\left(X_{n}^{-}\right) \notin \overline{\mathscr{C}}$.

3. The condition $\lim \inf _{\tau \in T} \min \left\{\int X_{\tau}^{+}, \int X_{\tau}^{-}\right\}<\infty$ in Theorem 4 is necessary. Let $\left(\xi_{n}\right)$ be i.i.d. r.v.s, $P\left(\xi_{n}=1\right)=P\left(\xi_{n}=-1\right)=1 / 2, X_{n}=\sum_{1}^{n} \xi_{i}, \mathscr{F}_{n}=\sigma\left(\xi_{1}, \ldots, \xi_{n}\right)$. Then, $\left(X_{n}\right)$ is a martingale, hence a pramart. It is well known that $\lim \sup _{n} X_{n}=\infty$ a.s. Hence, $\left(\left|X_{n}\right|\right) \notin \mathscr{E}$ 
and, by Theorem $3,\left(\left|X_{n}\right|\right)$ is not a pramart. It is easy to see that, in this example,

$$
\lim \inf _{\tau \in T} \min \left\{\int X_{\tau}^{+}, \int X_{\tau}^{-}\right\}=\frac{1}{2} \lim _{n} \int\left|X_{n}\right|=\infty .
$$

4. The set $\overline{\mathscr{C}}$ is larger than $\mathscr{C}$. Let $\left(Y_{n}\right)$ be i.i.d r.v.s, $P\left(Y_{n}=2\right)=P\left(Y_{n}=-1\right)=$ $P\left(Y_{n}=0\right)=1 / 3$. Let $X_{n}=3^{n} \prod_{1 \leq i \leq n} Y_{i}, \mathscr{F}_{n}=\sigma\left(Y_{1}, \ldots, Y_{n}\right)$. Then $\left(X_{n}\right)$ is a real-valued martingale. Let $\sigma=\inf \left\{n \geq 1, Y_{n}=0\right\}$. Clearly, $\sigma \in T_{f}$ and for any $\sigma \leq \tau \in T_{c}$, $\int_{(\tau<\infty)}\left|X_{\tau}\right|=0$. Hence, $\left(X_{n}\right) \in \overline{\mathscr{C}}$. Now let $\tau=\inf \left\{n \geq 1, Y_{n}=-1\right\}$. Then $\tau \in T_{f}, \int\left|X_{\tau}\right|=$ $\sum_{n} 6^{n}\left(P\left(Y_{1}=2\right)\right)^{n} / 2=\infty$, and $\left(X_{n}\right) \notin \mathscr{C}$.

\section{REFERENCES}

1. D. G. Austin, G. A. Edgar, and A. Ionescu Tulcea, Pointwise convergence in terms of expectations, Z. Wahrsch. Verw. Gebiete 30 (1974), 17-26.

2. A. Bellow, Uniform amarts: a class of asymptotic martingales for which strong almost sure convergence obtains. Z. Wahrsch. Verw. Gebiete 41 (1978), 177-191. $602-606$

3. A. Bellow and A. Dvoretzky, On martingales in the limit, Ann. Probab. 8 (1980), $340-346$

4. Y. S. Chow, Convergence theorems of martingales, Z. Wahrsch. Verw. Gebiete 1 (1963),

5. Y. S. Chow, H. Robbins, and D. Siegmund, Great expectations: The theory of optimal stopping (Houghton Mifflin, 1971).

6. Y. S. Chow and H. Teicher, Probability theory. Independence, interchangeability, martingales (Springer, 1978).

7. G. A. Edgar and L. Sucheston, Amarts: a class of asymptotic martingales. A. Discrete parameter, J. Multivariate Anal. 6 (1976), 193-221. $357-361$.

8. L. Egghe, Strong convergence of pramarts in Banach spaces, Canad. J. Math. 33 (1981),

9. L. Egghe, On sub- and superpramarts with values in a Banach lattice, Measure theory, Oberwolfach 1981, Lecture Notes in Mathematics 945 (1981), 353-365.

10. L. Egghe, Stopping time techniques for analysts and probabilists, London Mathematical Society Lecture Note Series 100 (Cambridge University Press, 1984).

11. N. E. Frangos, On convergence of vector valued pramarts and subpramarts, Canad. J. Math. 37 (1985), 260-270.

12. N. Ghoussoub, Orderamarts: A class of asymptotic martingales, J. Multivariate Anal. 9 (1979), 165-172.

13. N. Ghoussoub and L. Sucheston, A refinement of the Riesz decomposition for amarts and semiamarts, J. Multivariate Analysis 8 (1978), 146-150.

14. A. Gut, A contribution to the theory of asymptotic martingales, Glasgow Math. J. 23 (1982), 177-186.

15. A. Gut and K. D. Schmidt, Amarts and set function processes, Lecture Notes in Mathematics 1042. (Springer, 1983).

16. H. Heinich, Convergence des sous-martingales positives dans un Banach réticule, $C . R$. Acad. Sci. Paris Sér. A-B 286 (1978), A279-280.

17. U. Krengel and L. Sucheston, On semiamarts, amarts, and processes with finite value, Probability on Banach Spaces, Advances in Probability and Related Topics 4 (1978), 197-266.

18. K. Krickeberg, Convergence of martingales with a directed index set, Trans. Amer. Math. Soc. 83 (1956), 313-337.

19. A. Millet and L. Sucheston, Characterizations of Vitali conditions with overlap in terms of convergence of amarts, Canad. J. Math. 31 (1979), 1033-1046.

20. A. Millet and L. Sucheston, Convergence of classes of amarts indexed by directed sets, Canad. J. Math. 32 (1980), 86-125. 
21. A. G. Mucci, Limits for martingale-like sequences, Pacific J. Math. 48 (1973), 197-202.

22. A. G. Mucci, Another Martingale convergence theorem, Pacific J. Math. 64 (1976), 539-541.

23. J. Neveu, Discrete-parameter martingales (North-Holland, 1975).

24. K. D. Schmidt, The lattice property of uniform amarts, Ann. Probab. 17 (1989), 372-378.

25. H.-U. Schwarz, Banach lattices and operators, Teubner-Texte zur Mathematik 71 (B. G. Teubner, 1984).

26. M. Staby, Convergence of positive subpramarts and pramarts in Banach spaces with unconditional bases, Bull. Polish Acad. Sci. Math. 31 (1983), 75-80.

27. M. Słaby, Strong convergence of vector-valued pramarts and subpramarts, Probab. Math. Statist. 5 (1985), 187-190.

28. M. Talagrand, Some structure results for martingales in the limit and pramarts, Ann. Probab. 13 (1985), 1192-1203.

29. Z. P. Wang, The lattice properties of martingale-like sequences, Acta Math. Sinica 30 (1987), 355-360.

30. Z. P. Wang, Local convergence of martingale-like sequences, Chinese Ann. Math. Ser. A 9 (1988), 203-207.

31. Z. P. Wang and X. H. Xue, On convergence of vector-valued mils indexed by a directed set, Almost everywhere convergence (Columbus, Ohio, 1988) ed. G. Edgar, and L. Sucheston, (Academic Press, 1989), 401-416.

32. X. H. Xue, On convergence of pramarts in Banach spaces, Bulletin of Chinese Science 29 (1984), 1280.

33. X. H. Xue, On convergence of subpramarts and games which become better with time, $J$. Theoret. Probab. 4 (1991), 605-623.

34. Y. Yamasaki, Another convergence theorem of martingales in the limit, Tôhoku Math. J. 33 (1981), 555-559.

DEPARTMENT OF STATISTICS

East China Normal University

SHANGHAI 200062

China
DePartMENT OF Statistics

COLUMBia UNIVERSITY

NEW YORK

NY 10027

U.S.A. 